\title{
INFLUENCE OF FREEZING AND STORING CHERRY FRUIT ON ITS NUTRITIONAL VALUE
}

\author{
Elena Vasylyshyna ${ }^{\varpi}$ \\ Faculty of Engineering and Technology, Uman National University of Horticulture \\ Institutska 1, Uman, Cherkasy region, 20305 Ukraine
}

\begin{abstract}
Background. Cherries are a valuable dietary raw material and possess medicinal properties. Considering the nutritional, medical and vitamin value of cherry fruits, the purpose of this research was to produce a scientific justification for preserving the quality of cherry fruits using different freezing methods.

Material and methods. To do this, cherry fruits from the Lotovka (Cerasus vulgaris) variety were frozen in various ways: packed in polyethylene bags (control); previously suspended in a $20 \%$ sugar solution and packing frozen cherry in polyethylene bags; suspended in a 20\% sugar solution with the addition of $4 \%$ ascorutin and frozen followed by pre-packaging in polyethylene bags; cherry fruits were frozen in a $20 \%$ sugar solution in plastic cups of $0.25 \mathrm{~cm}^{3}$; they were also frozen in a $20 \%$ sugar solution with the addition of $4 \%$ ascorutin in plastic cups. The frozen products were stored at a temperature not higher than $-18^{\circ} \mathrm{C}$ for up to 6 months. Result. Studies have shown the appropriateness of freezing cherry fruits, particularly in a $20 \%$ sugar solution with the addition of $4 \%$ ascorutin. The advantages of these fruits are in ascorbic acid preservation in 1.5 times and reduction of tanning and coloring substances only by $27 \%$, soluble solids to $7 \%$, sugars $-4 \%$, acids $12 \%$, in tasting evaluation of 5 points.

Conclusions. Frozen cherry fruits in a $20 \%$ sugar solution with the addition of $4 \%$ ascorutin can be used in dietary nutrition for patients with cardiovascular diseases.
\end{abstract}

Key words: frozen cherry fruits, soluble solids, ascorbic acid, tanning and coloring substances

\section{INTRODUCTION}

Cherry fruits are a good combination of sugars and acids with a pleasant color and taste. They are widely used as fresh and processed products. Juice, fruit compote, jelly, fruit drinks and syrup are prepared, jam may be made from them, and different extracts, liqueurs and fruit water are also made from these fruits. Berries are widely used in the confectionery industry, where they are added to various dishes. They are delicious fresh and as a dessert, and unusual in combination with ice cream, jelly, or in cocktails (Chernozubenko, 1993; Gudkovsky, 2001; Kuyan, 2004).
The widespread use of cherry juice is due to their being easily digested and having a high vitamin value. For the production of juices and fruit compotes, cherry fruits with an intense color in their pulp form and low acidity are used (Chernozubenko, 1993).

Useful properties of cherries are explained by their composition. The cherry contains sugars $(15 \%)$, of which glucose, organic acids, vitamins B1, B2, C, $\mathrm{P}, \mathrm{PP}$, carotene, folic acid, pectic substances, mineral salts of iron, copper, manganese, potassium, iodine, phosphorus, magnesium and cobalt dominate. These 
biologically active substances are responsible for the medicinal properties of cherry fruits in combatting many diseases (Gudkovsky, 2001; Zhurba, 1987).

Research has shown that cherries revitalize the cardiovascular system, as they contain a compound of $\mathrm{P}$-vitamin complex (more in darker varieties) and they are evenly located throughout the pulp, thus making them easily available for absorption.

Anthocyanins pigments have good digestibility, as they are distributed throughout the pulp of the fruit, so cherries with a red color are very useful for the brain and improving the memory. Research has shown that cherry fruits contain ellagic acid, which blocks the development of malignant tumors and prevents the growth and development of cancer cells (Zhurba, 1987).

Coumarin, which is found in cherries, is a substance that reduces blood clotting. Due to this property it is used as a means of preventing certain complications of arterial atherosclerosis (Tsyriapin et al., 1999; Zhurba, 1987).

The successful combination in cherry fruits of vitamins $\mathrm{C}, \mathrm{B} 1, \mathrm{~B} 6$, iron, magnesium, cobalt and pigments prevents and treats anemia.

The cherry contains blood-forming minerals: iron, copper and cobalt, which allow its use in anemia. The presence of iodine $(330 \mathrm{mcg})$ in cherries is of great importance. A lack of iodine leads to the development of goiter, so cherries might prevent this serious abnormality. Cherries also contribute to the removal of oxalic acid from the body and this prevents the formation of stones and salts in different organs - the kidneys, bladder, liver and also in the joints (Chernozubenko, 1993; Tsyriapin et al., 1999; Zhurba, 1987).

Pectin substances contribute to the removal of heavy metals, radionuclides and nitrogenous wastes from the body. Fruit pulp of the cherry contains bactericidal substances. Cherry juice is drunk in cases of mental diseases, arthritis; it quenches thirst and improves the appetite. Regular consumption of cherries lowers the risk of gout.

Ascorbic acid in combination with P-vitamin, tanning substances and pigments of cherries tone up and fasten blood capillaries, lower the blood pressure and increase the body's resistance to adverse factors. Consumption of cherry fruits reduces the frequency and reduces the impact of heart attacks (Chernozubenko, 1993).
Therefore, because of its beneficial properties, cherry fruits are widely used in folk medicine as a dietary product which improves the appetite and digestion, quenches thirst, and has an antiseptic and expectorant effect. Cherry fruits are used with milk in the treatment of arthritis. Their pulp and juice has an expectorant effect in the inflammation of the airways.

Thus, cherry fruits are a source of soluble organic substances, vitamins and trace elements that have healing properties. However, they may only be used around maturation, a time period limited to 10 to 15 days. Therefore, one way to extend the period is fruit freezing.

Freezing as a way of preserving the original flavor and biologically active substances of the cherry as much as possible. The main requirement for freezing is to provide conditions under which soft cherry fruits are not deformed, their integrity is preserved, the possibility of congelating individual fruits is excluded, a free-running frozen product is produced that is easy to pack and process (Arasimovich, 1996; Artiomova and Makarina, 2006).

The quality of berry freezing is influenced by the conditions in which the product is obtained. Therefore, there are the following methods of freezing: mass freezing in pieces, freezing of berries in a sugar syrup, including that with the addition of substances which preserve the quality of the product.

The more concentrated the sugar syrup, the slower the freezing process. Instead of water, during the preparation of syrup, one might use fresh juice and add biologically active substances such as ascorutin (Gudkovsky, 2001; Tsyriapin et al., 1999).

The value of ascorutin is that its ingredients are ascorbic acid and rutin - biologically active substances that potentiate each other's effects, whereby the inhibition of peroxidation processes is achieved, thus preserving the high quality of the product. Furthermore, ascorbic acid and rutin are antioxidants recognized as synergists with respect to each other. This synergism consists in the fact that rutin retards oxidation of ascorbic acid and enhances its antioxidant properties. At the same time, ascorbic acid increases the activity of rutin. As a result, the accumulation of peroxide compounds that cause the berries to brown is inhibited (Gudkovsky, 2001). 
Given the nutritional and therapeutic value of cherry fruits, the purpose of this work was to provide a scientific justification for preserving the quality of cherry fruits using different freezing methods.

\section{MATERIAL AND METHODS}

The studies were carried out during 2012-2013 with cherry fruits of the variety Lotovka (Cerasus vulgaris) grown in the Central region of Ukraine. The variety is zoned in the forest steppe, Polesye and Western Ukraine. The fruits weighed $4-5 \mathrm{~g}$, are flattened round in shape, dark red in colour, with juicy flesh and a sour-sweet taste. They ripen in late June - early July (Kuyan, 2004).

The studies used ascorutin - a vitamin preparation which consists of rutin and ascorbic acid (1:1).

Research variants:

- frozen cherry fruits packed in polyethylene bags (control)

- cherry fruits previously submerged in a $20 \%$ solution of sugar and frozen packed in polyethylene bags

- cherry fruits previously suspended in a $20 \%$ sugar solution with the addition of $4 \%$ ascorutin and frozen, packed in polyethylene bags

- cherry fruits frozen in a $20 \%$ solution of sugar in plastic cups of $0.25 \mathrm{~cm}^{3}$

- frozen cherry fruits in a $20 \%$ sugar solution with the addition of $4 \%$ ascorutin in plastic cups of $0.25 \mathrm{~cm}^{3}$.

Preparation of cherry fruits included sorting, inspection, washing, removing remaining moisture with filter paper. The fruits were held in sugar syrup with added ascorutin for 30 minutes. The fruits were then frozen in chambers packed in $500 \mathrm{~g}$ portions in polyethylene bags or frozen in sugar syrup with the addition of ascorutin in $200 \mathrm{~g}$ portions in cups with thermoformed plastic in Calex chambers at a temperature of $-22-24^{\circ} \mathrm{C}$. The frozen products were stored at a temperature no higher than $-18^{\circ} \mathrm{C}$ for up to 6 months.

Quality assessment of the cherry fruit was conducted in stages: before and after freezing - for one day or two, four and six months of storage and with reference to the following indicators: mass fraction of soluble solids - by the refractometric method (Naichenko, 2001), invert sugars - by the ferricyanide method (Naichenko, 2001), titrating acids - by titration with alkali
(GOST 25555.0-82), ascorbic acid - by the iodometric method (Naichenko, 2001), tanning and coloring substances - by Neubauer and Leventhal's method (Naichenko, 2001), catalase activity by Pochinok (1976). The evaluation was performed on a 5-point scale (Guidelines..., 1984).

Statistical data processing and correlation was performed (Dospehov, 1979) using the "Excel 2000" program.

\section{RESULTS AND DISCUSSION}

According to the studies, fresh cherry fruits contained $15 \%$ soluble solids, including $8 \%$ invert sugars, $1.5 \%$ acids, $1.5 \%$ of tanning and coloring substances and 20 $\mathrm{mg} / 100 \mathrm{~g}$ of ascorbic acid.

During the deep freezing and storage of cherry fruits in polyethylene bags there was a decrease in the content of soluble solids to $7-17 \%$ (Fig. 1), including a decrease in invert sugars of $4-5 \%$. Due to osmotically diffuse processes in cherry fruits frozen in sugar solutions, there was a $2 \%$ increase in soluble solids content.

The acidity of frozen cherry fruits during the whole storage period was reduced (Fig. 2 ) - by $12-20 \%$. The smallest loss of organic substances was identified in cherry fruits previously sustained in a $20 \%$ sugar solution and with the addition of $4 \%$ ascorutin, which is associated with the inhibition of peroxidation processes.

In general, these were the best indicators of preserving organic acids of cherry fruits frozen in $20 \%$ sugar solution with the addition of $4 \%$ ascorutin.

During their 6 months storiage, the frozen fruits' ascorbic acid content decreased in the controls by $56 \%$, whereas in cherry fruits previously sustained in sugar syrup with the addition of $4 \%$ ascorutin frozen in plastic cups, this fell by 35\% (Fig. 3), stemming from the fact that ascorutin contains L-ascorbic acid.

Product quality is characterized by the content of tanning and coloring substances. Cherry fruits are valuable in these substances, the content of which is $1.5 \%$ (Fig. 4 ).

During the whole period of storing frozen cherry fruits in pieces and packaged in polyethylene bags, a decrease in tanning and coloring substances up to $30-39 \%$ was recorded, whereas in fruits frozen in sugar syrup and with the addition of ascorutin in plastic 

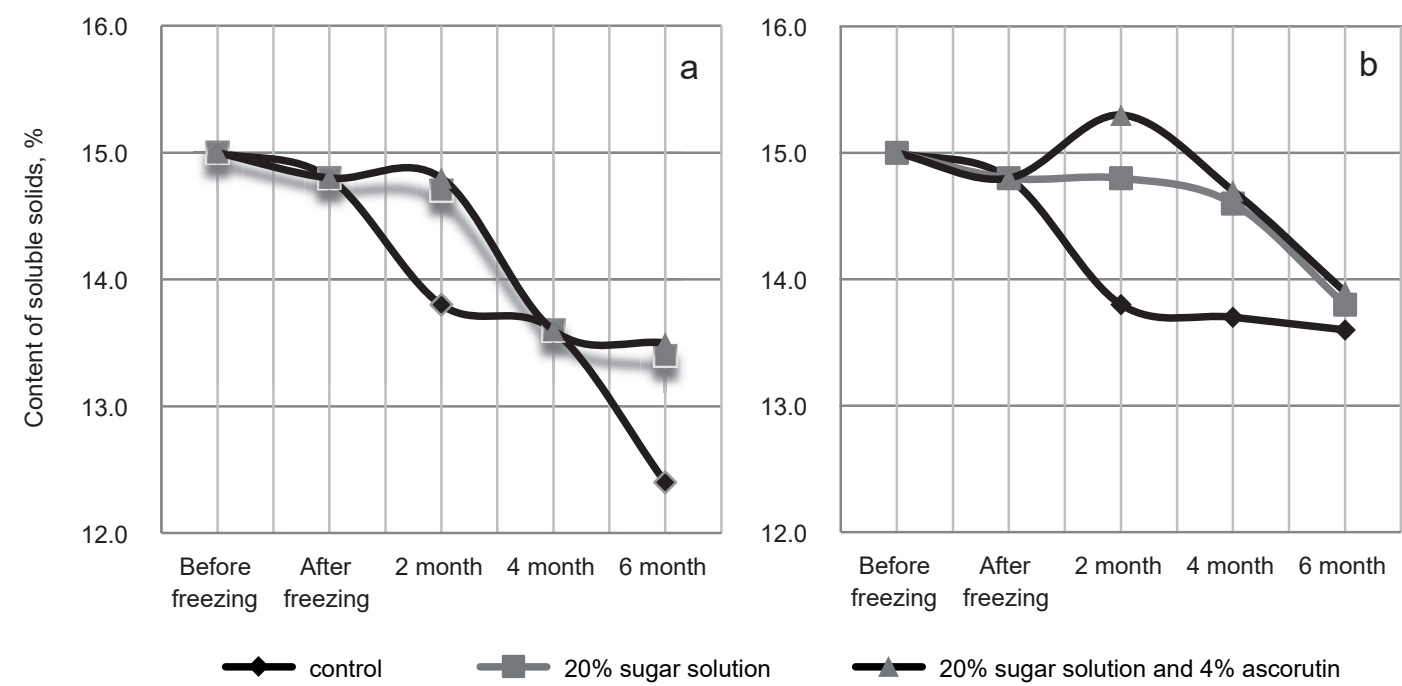

Fig. 1. Dynamics of content of soluble solids in cherry fruits at freezing: $a$ - in polyethylene bags, $b-$ plastic cups
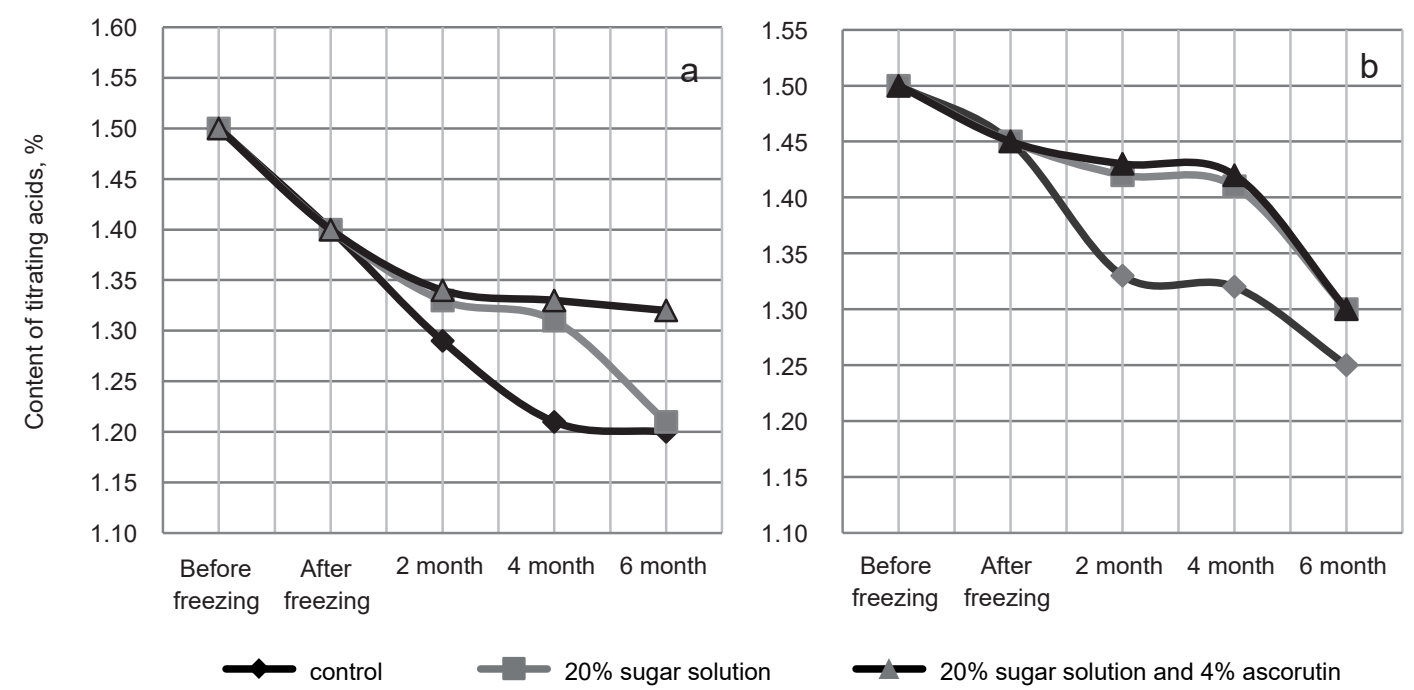

Fig. 2. Dynamics of content of titrating acids in cherry fruits at freezing: $a$ - in polyethylene bags, $b-$ plastic cups

cups, this rose by $27 \%$. The content of tannins and coloring substances in frozen fruits remained at a level of $0.92-1.1 \%$. Changing the content of tannins and coloring substances during the storage of frozen cherry fruits is caused by peculiarities in the behavior of the most easily oxidized leucoanthocyanins and catechins.

Metabolism in cherry fruits depends on the activity of enzymes. When freezing, due to the destruction of enzymes, the balance and coordination of individual reactions, their synchronicity breaks down, and the products of incomplete oxidation of energy substrates are accumulated, thus deteriorating the quality of the fruits.

Cherry fruit quality in the process and after defrosting is determined by the activity of redox enzymes, which include catalase. We found that the activity of the enzyme catalase in cherry fruits is $2.5 \mathrm{~mol} / \mathrm{min}$. 

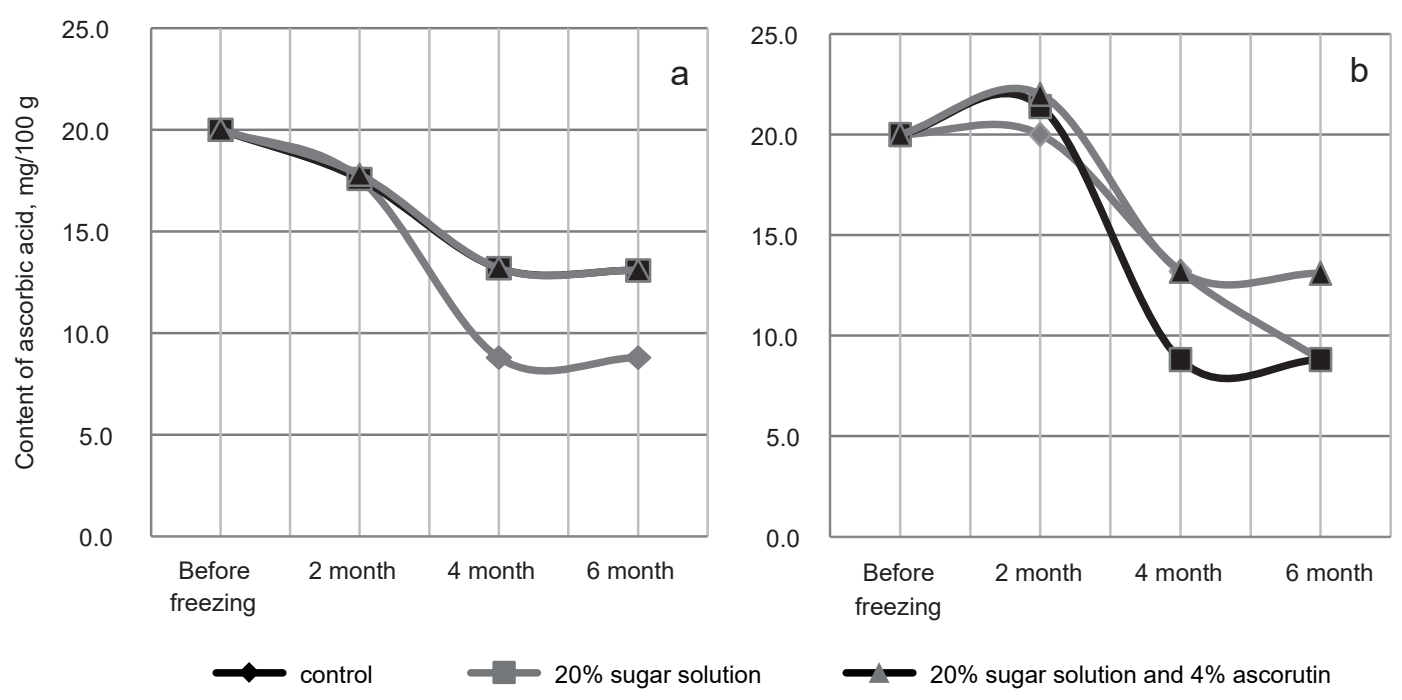

Fig. 3. Changing content of ascorbic acid in cherry fruits during freezing and storage: a - polyethylene bags, $\mathrm{b}$ - plastic cups
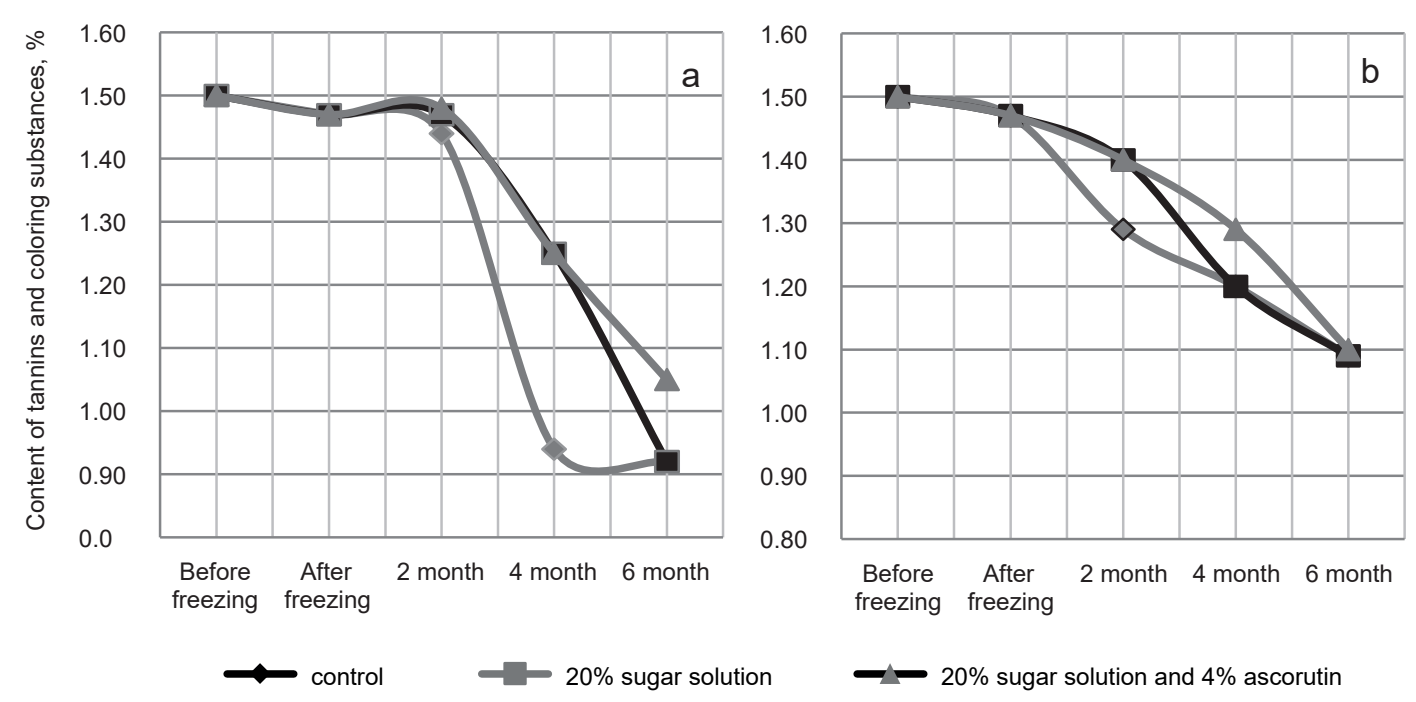

$-20 \%$ sugar solution and $4 \%$ ascorutin

Fig. 4. Changing content of tannins and coloring substances in cherry fruit during freezing and storage: $\mathrm{a}$ - polyethylene bags, $\mathrm{b}$ - plastic cups

After six months of storage, there was a 2-fold decrease in fruits of all varieties.

This indicates that the progress of oxidation-reduction reactions in fruits after defrosting affects their quality changes.

As in cherry fruits, there is synergism of ascorbic acid with flavonoids, having P-vitamin activity. This reinforces its action, protects against damage and prolongs its safety due to the inactivation of ascorbate oxidase due to blocking the copper within it (Gudkovsky, 2001).

We found a correlation coefficient between the content of tannins, coloring substances and ascorbic acid $-r=0.85$ and a equation of regression for correlation dependence $y=19.2 x-9.1$ in which, depending on the content of tannins and coloring substances in cherry fruits, ascorbic acid content may be determined (Fig. 5). 


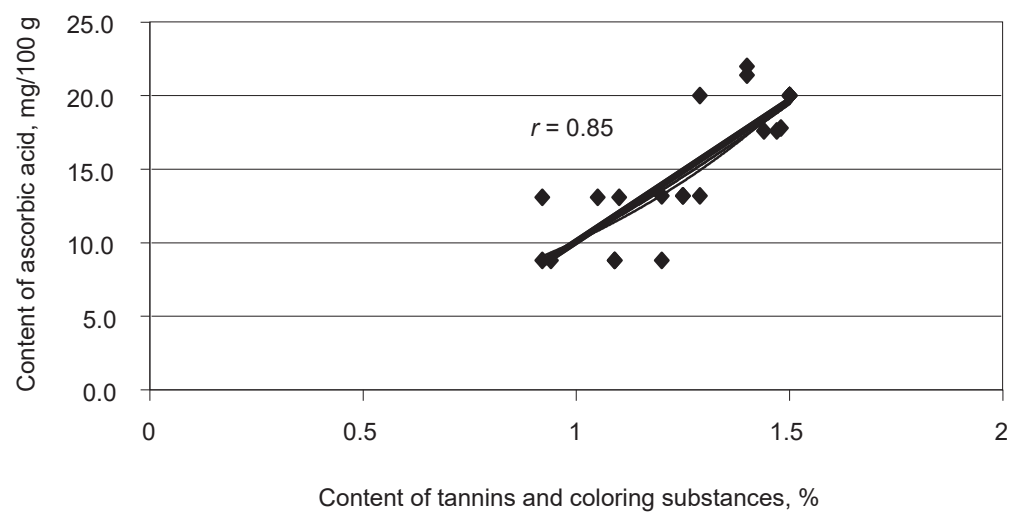

Fig. 5. Point schedule and theoretical line of regression of straight dependence between the content of tannins and coloring substances and ascorbic acid

Tasting assessment of frozen cherry fruits were estimated at 4.2-4.3 points. By color and appearance of cherry fruits frozen in a $20 \%$ sugar solution and with the addition of ascorutin received 5 points.

\section{CONCLUSIONS}

Experiments showed the expediency of freezing cherry fruits especially in a $20 \%$ sugar solution with the addition of $4 \%$ ascorutin. The benefits of such fruits are as follows: preserving ascorbic acid in $20 \%$, content reduction of tannins and coloring substances only by $27 \%$, soluble solids by $7 \%$, sugars by $4 \%$, acids by $12 \%$ at tasting score in 5 points.

Frozen cherry fruits in a $20 \%$ sugar solution with the addition of $4 \%$ ascorutin can be used in the dietary nutrition for patients with cardiovascular diseases.

\section{REFERENCES}

Arasimovich, V. V. (1996). Studying patterns of variability of carbohydrates of fruits and vegetables and ways to use them. Chisinau: Moldova State Publishing House.

Artiomova, Y. N., Makarina, N. V. (2006). Quality of the dietary jelly made from red currant of the variety Marmeladnitsa. Storag. Process. Agric. Raw Mater., 12, 39-41.
Chernozubenko, N. K. (1993). Determination of suitability of new varieties of black currants and cherries for storage and processing. Thesis abstract on scientific degree of Candidate of Agricultural Sciences: Specialty 05.18.03 "Primary processing and storage of crop growing products". Kyiv.

Dospehov, B. A. (1979). Technique of field experience with the fundamentals of statistical processing studies. Moscow: Kolos.

Guidelines for research with frozen fruits, berries and vegetables (1984). Moscow: Agricultural Sciences.

Gudkovsky, V. A. (2001). Antioxidant (healing) properties of fruits and berries and progressive methods of their storage. Storag. Process. Agric. Raw Mater., 4, 13-15.

GOST 25555.0-82. Products of processing fruits and vegetables. Methods for determination of titratable acidity (1983). Moscow: Publishing House of Standards.

Kuyan, V. G. (2004). Special fruit growing: Textbook. Kyiv: World.

Naichenko, V. M. (2001). Practicum on technology of storage and processing of fruits and vegetables (pp. 158162). Kyiv: Pupil.

Pochinok, K. N. (1976). Methods of biochemical analysis of plants. Kyiv: Scientific Thought.

Tsyriapin, V. I., Vankhanin, V. D., Smiliansky, B. L. (1999). Food hygiene with basics of nutritiology: Textbook. Kyiv: Health.

Zhurba, M. A. (1987). Biologically active substances in cherry fruits. Fruit Veget. Household, 7, 56. 